\title{
McMaster Virtual Design Projects
}

\author{
$\begin{array}{lll}\text { A. Spence } & \text { B. Ruberto } & \text { C. Parsisson }\end{array}$ \\ adspence@mcmaster.carubertb@mcmaster.ca parsiscp@mcmaster.ca \\ McMaster University, Department of Mechanical Engineering
}

\begin{abstract}
This paper describes several 2003-2004 projects using virtual design tools at McMaster University. Students begin by repeat modeling of a part from first year. New projects were to develop a $3 D$ CAD tool for quickly updating new home kitchen design, and to apply CAD to Formula SAE racecar cockpit design. An anthropomorphic model was included to verify reach and visibility. In both cases, stereo display was used to improve the $3 D$ visualization, and comparison was made with actual construction. The third project was to explore online CAD animation of a FARO measuring arm.
\end{abstract}

\section{Introduction}

For graduating engineering students, particularly in the mechanical and civil disciplines, 3D modeling abilities are desired by prospective employers. In this paper, several 2003-2004 projects in the use of Computer Aided Design (CAD) and related virtual design tools are described.

Students review by modeling a part from first year. This provides a common starting point, and an opportunity to present introductory material on solid modeling and computer graphics.

Next, the paper describes two recent undergraduate projects on virtual design. The first project was a kitchen design for a new home, and the second project was cockpit design for the McMaster Formula SAE racecar. Each project was carried out using Autodesk Inventor based 3D solid models. An anthropomorphic model was included to verify scale, reach, and visibility. Two different styles of stereo eyewear are used to create an affordable 3D immersive environment. Comparison is made with actual construction.

The third project was telemetry based CAD animation of a FARO measuring arm. This was implemented very quickly using Visual Basic for Applications (VBA) within Inventor.
The remainder of the paper is organized as follows. In Section 2, the introductory curriculum and review assignment are briefly described. In Section 3, the new home kitchen project is described. Section 4 describes the Formula SAE cockpit project. Stereo display technology is presented in Section 5. Section 6 describes the online FARO arm animation project. An overall discussion of the projects is contained in Section 7, followed by a summary in Section 8 .

\section{Modeling Review}

The McMaster first year design curriculum has recently been revised [1]. During the transition, there will remain students who reach final year with neither formal 3D CAD education nor experience, and hence a common introduction is required.

The common introduction consists of a brief review of 2D sketching, 3D Constructive Solid Geometry (CSG) and Boundary Representation modelers. Relevant computer graphics display technology is presented initially, and reinforced through projects. To provide a bridge, students are assigned the task of modeling a part from the first year course (Figure 1). They are provided with the 2D paper space drawing, and asked to create the 3D solid. Each face of the part is to be coloured differently. To further check their work, characteristics such as surface area, volume, etc. are verified [1]. As a further aid, rapid prototype models of the parts, with the same coloured faces, are also available (Figure 2).

\section{New Home Kitchen Project}

\section{Background}

For 2003, it was estimated that there were 217,800 new housing starts in Canada [2]. Contemporary purchasers desire a custom design to suit personal taste, but this can be difficult to achieve. At the early stage, purchasers have difficulty visualizing how their concept will look when complete. The inexperienced purchaser is likely to 


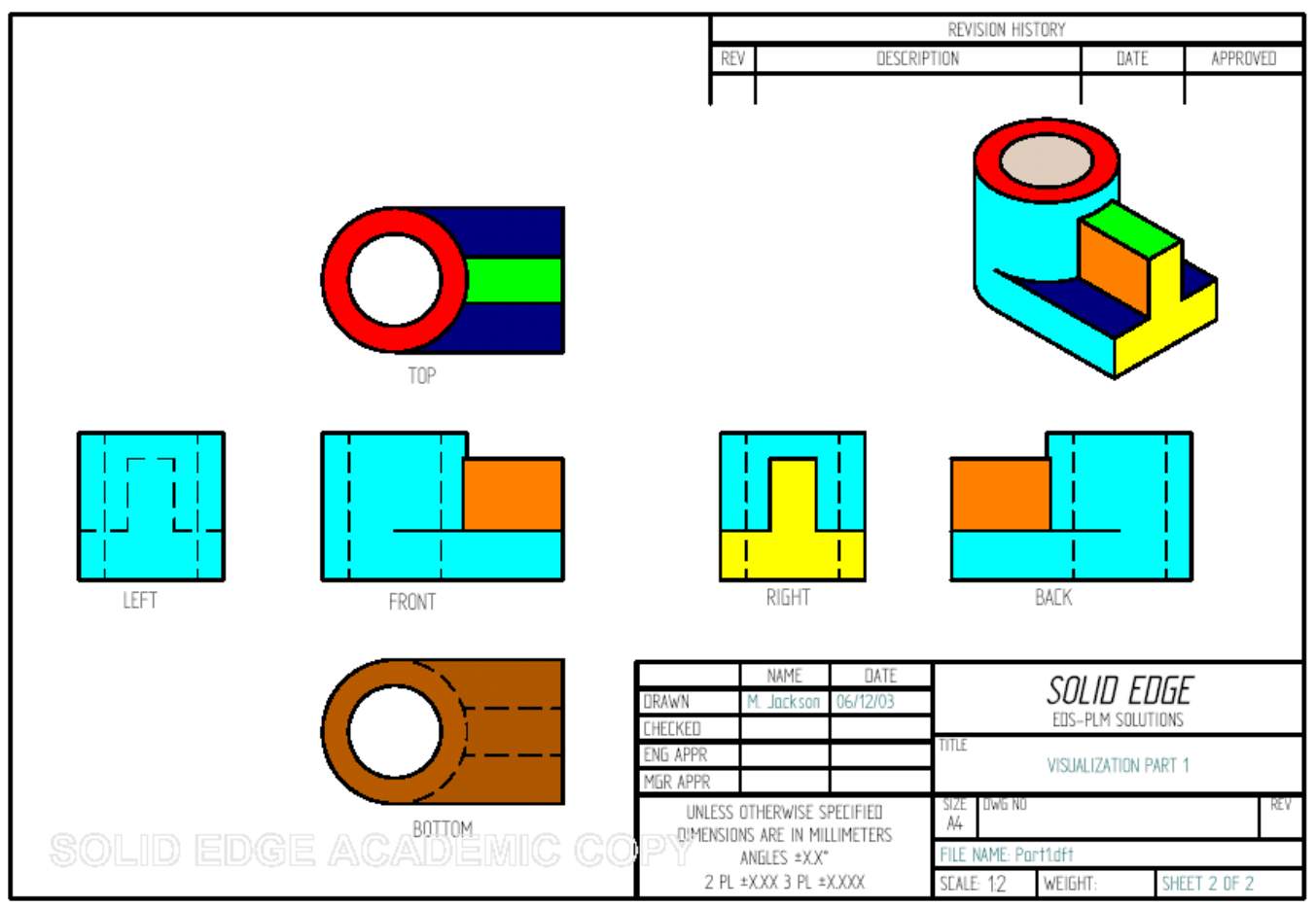

Figure 1. Review part with coloured faces

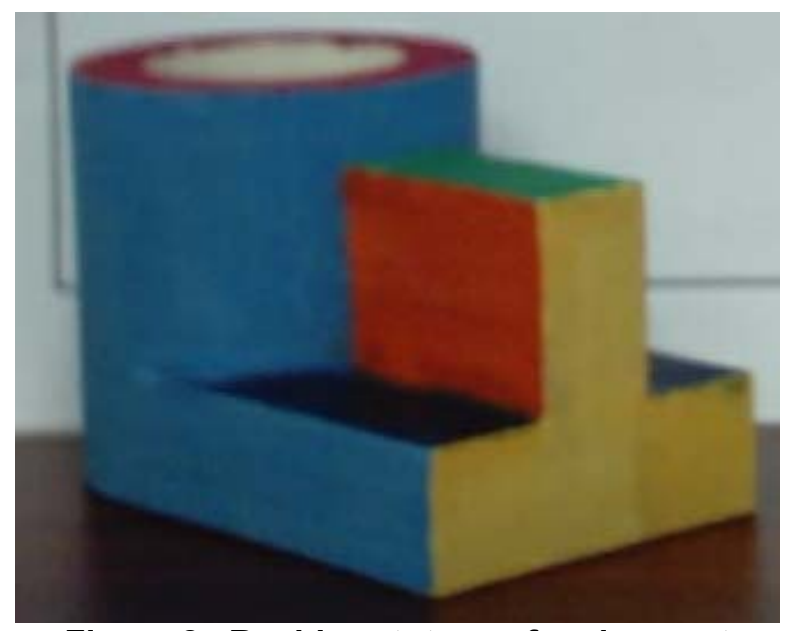

Figure 2. Rapid prototype of review part

overlook trade rules of thumb, building code requirements, and, without expert assistance, will likely be disappointed in the final result. Furthermore, the future owner will have difficulty adequately describing their wishes to the contractor, and, during actual construction, to tradespeople unfamiliar with the custom design. The result often significantly deviates from intent. This causes disappointment for the purchaser, extra cost for remedial correction, and disillusionment between the participants. Many of these difficulties can be solved by developing and using appropriate design and visualization software tools.

For this project, the students first reviewed existing software including IKEA Kitchen Planner [3] View22 [4], Autokitchen [5], and 20-20 Design [6]. The IKEA Kitchen Planner runs as a standalone program and can create a Bill of Materials for IKEA specific items. View22 employs a web client/server architecture, and has been server licensed for use by companies including GE Appliances, Sears, and Bob Vila. Both are products intended for free client home users, and rely on facetted displays without an 


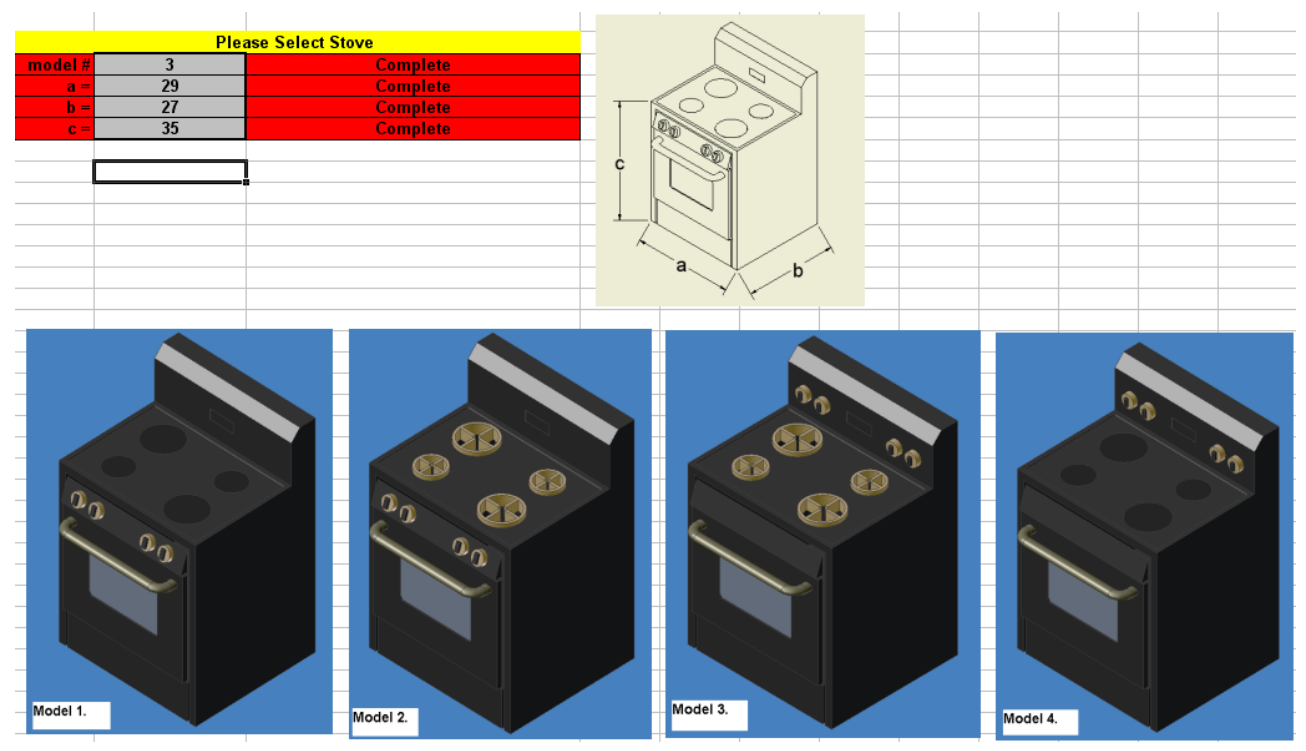

Figure 3. Excel stove iPart specification spreadsheet

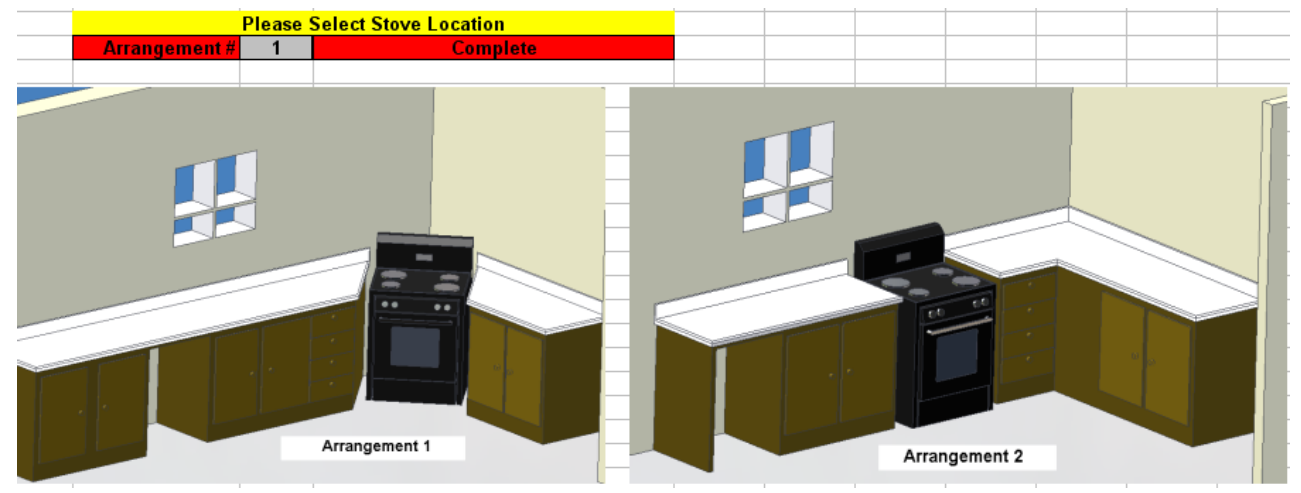

Figure 4. Excel stove assembly location spreadsheet

underlying solid model. Currently, neither product offers very high quality rendering or professional quality drawings. The intended use is for marketing as opposed to precision design. Autokitchen and 20-20 Design are more professional products, with purchase prices of less than CDN\$1000. Autokitchen is based on AutoCAD [7], and hence exact paper drawing creation is fully supported. The 20-20 Design product has built-in photorealistic rendering capabilities.

Because of the high per seat software licensing costs, and the slower computing speeds associated with desktop computers, the majority of the above packages depend on graphics toolkits such as SGI Open Inventor [8]. This is in contrast to the established direction in mechanical CAD, where preferred mechanism design [9] and Finite Element Analysis [10] add-ons are integrated with industry standard solid modelers. It is anticipated that civil and architectural CAD at all levels will eventually migrate to 3D solid models as well [11].

\section{Implementation}

The new home kitchen project was based on the Autodesk Inventor [12] 3D solid modeler. To investigate various design possibilities, representative cabinets and appliances were drawn as individual parts, and then added to an overall assembly. In order to change shapes and suppress features without the need for a library of files, the Inventor iPart capability was used. A corresponding Microsoft Excel spreadsheet table contained the changeable parameters for the part, including dimensions and toggles that specify whether or not a feature is computed or suppressed. Excel Visual Basic for Applications (VBA) macros simplified the user interface. The spreadsheet used to select the stove is shown in Figure 3. 


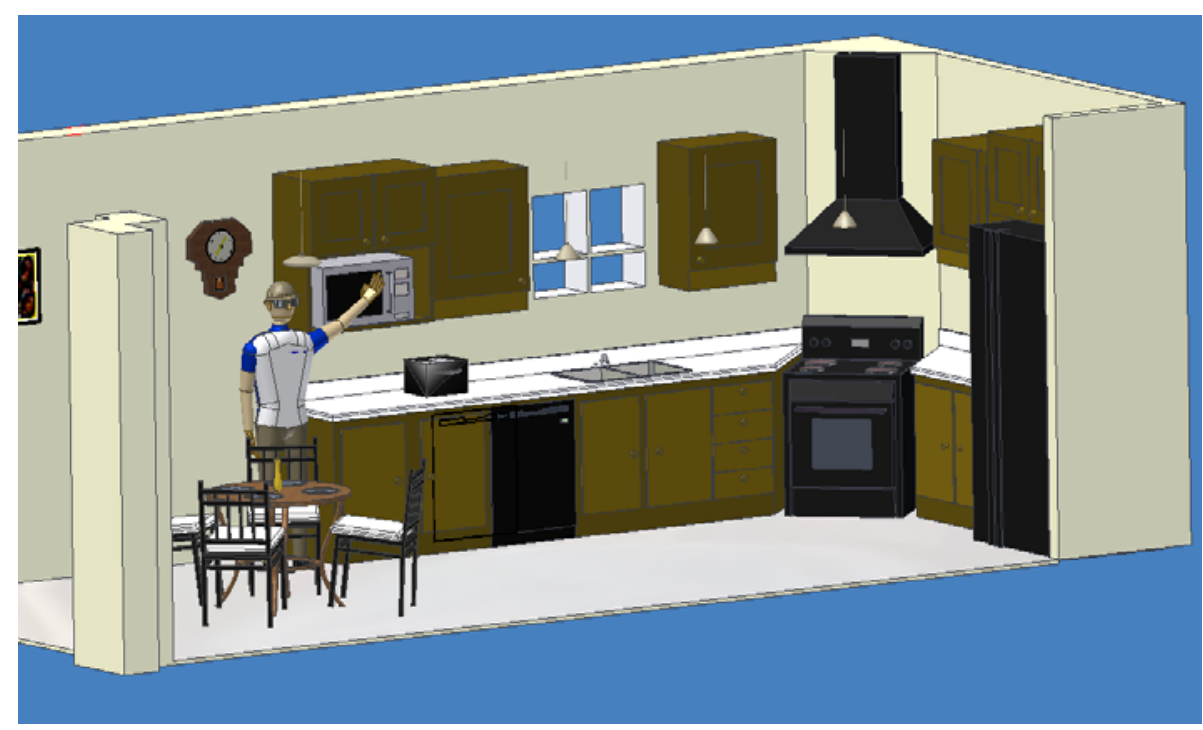

Figure 5. Inventor assembly including anthropomorphic model

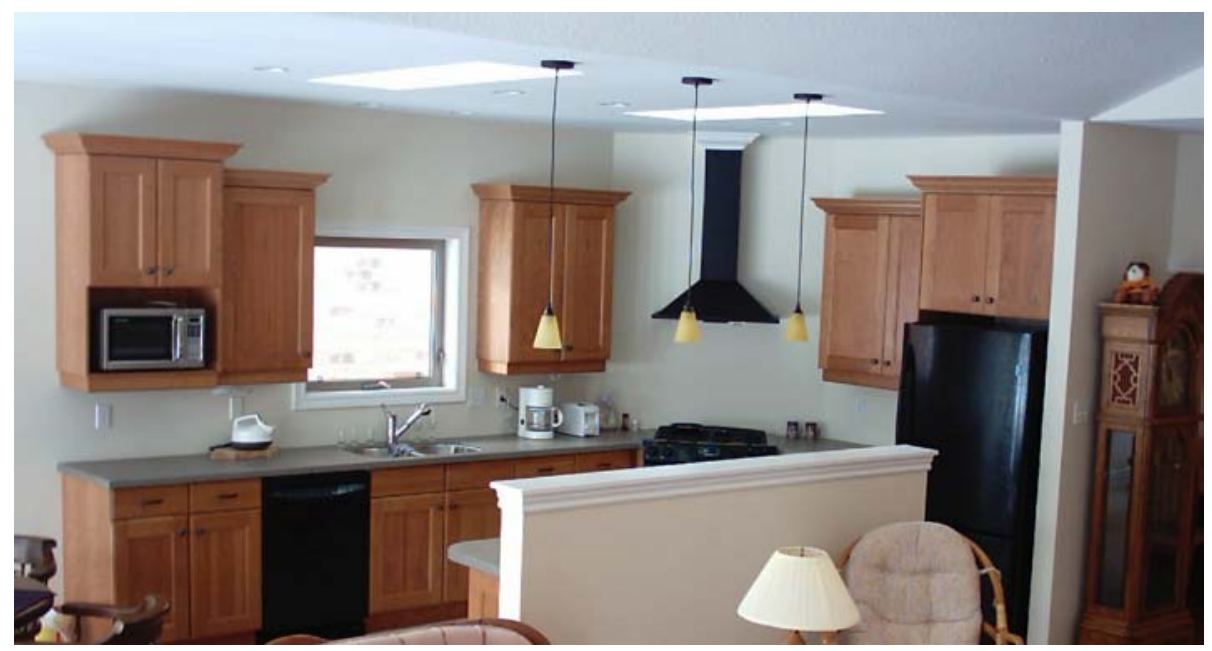

Figure 6. Actually constructed kitchen photograph

At the assembly level, the position of the stove can be specified by the user. The opening in the countertop and cabinets is automatically adjusted to suit (Figure 4). The complete Inventor kitchen model, including the iMike anthropomorphic model [13], is shown in Figure 5.

The CAD generated assembly images were used during discussions with both the kitchen cabinet designer, and with the general contractor. Of course, determining whether or not the images avoided any incidents of miscommunication and mistakes is impossible to tell. However, it is noted that, during construction, the builder actually nailed a printed copy to the future kitchen wall stud. A photograph of the actual constructed kitchen is shown in Figure 6. The fidelity with the CAD image is remarkable.

\section{Formula SAE Cockpit Project}

\section{Background}

Approximately 120 college and university student teams participate in the annual Formula SAE competition [14]. The race car must periodically be redesigned to satisfy competition rules, and virtual design tools can help to avoid mistakes. The project undertaken concentrated on cockpit ergonomics. Previous problems included important gauges and indicator lights being hidden by the steering wheel or driver's hands, and switches being located either where they were difficult to reach, or could inadvertently be actuated by the driver's legs, stopping the engine. 


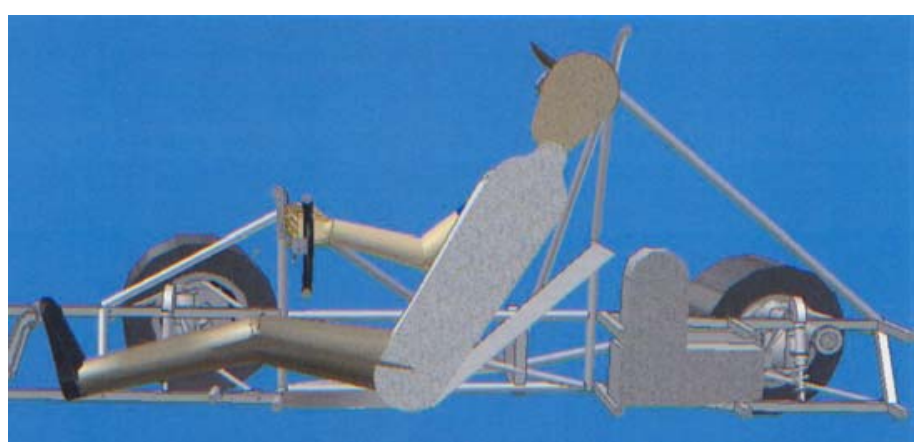

Figure 7. Formula SAE cockpit cross section, including iMike

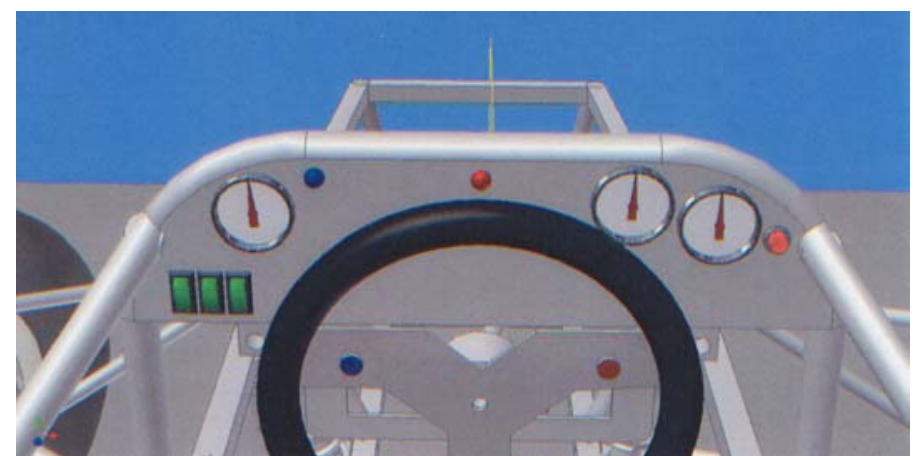

Figure 8. Formula SAE instrument panel CAD model

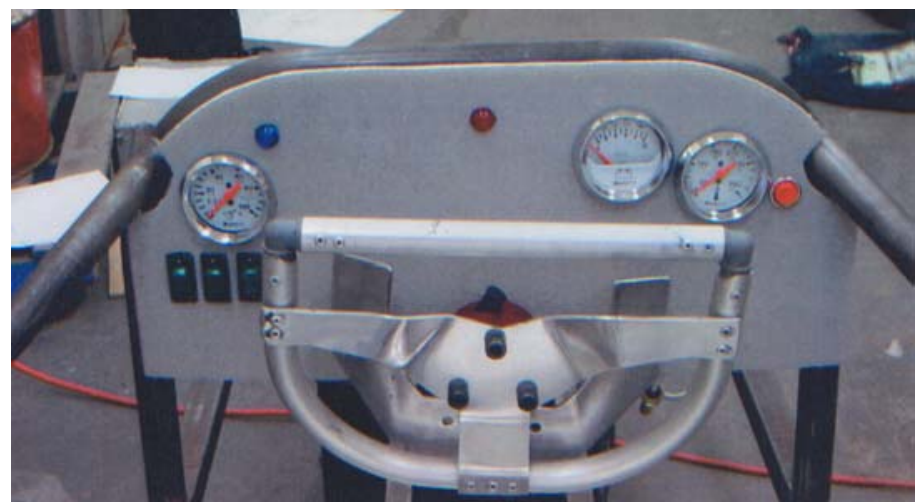

Figure 9. Actual Formula SAE instrument panel photograph

\section{Design Steps}

To research the problem, the Formula SAE team referred to texts on engineering design aesthetics [15] and human factors [16]. These books provided valuable references on proper placement of dials and gauges, and statistical information on human body size, reach force capabilities, etc. The iMike anthropomorphic model [13] was inserted into the proposed Autodesk Inventor race car cockpit (Figure 7). As with the new home kitchen, a linked Excel spreadsheet could be adjusted to model different drivers.

Significant effort was directed towards design of the instrument display panel, and in total six CAD based iterations were made. To best mimic actual use, the panel was viewed from the actual iMike eye position. Beginning at the left of Figure 8, the instruments included are the engine management switch, fuel pump switch, shift computer switch, oil pressure gauge, blue neutral light, red shift light, voltage gauge, temperature gauge, and starter switch. This same layout (with a slightly different steering wheel shape), can be seen in the first physical prototype (Figure 9).

Team members credited the virtual design approach with more rapid progress towards a physically workable cockpit layout. The Inventor CAD model, combined with iMike, helped to quickly establish correct seating and pedal placement. A nongeometric observation was the relative ease with which the modern "racing" white face gauges could be read compared to the more traditional black faces. 


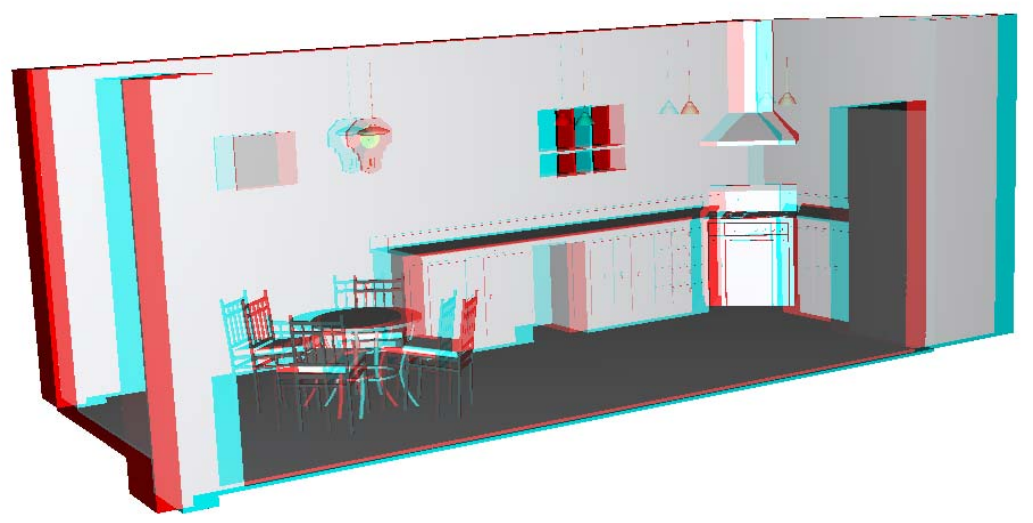

Figure 10. New home kitchen stereo red-blue anaglyph

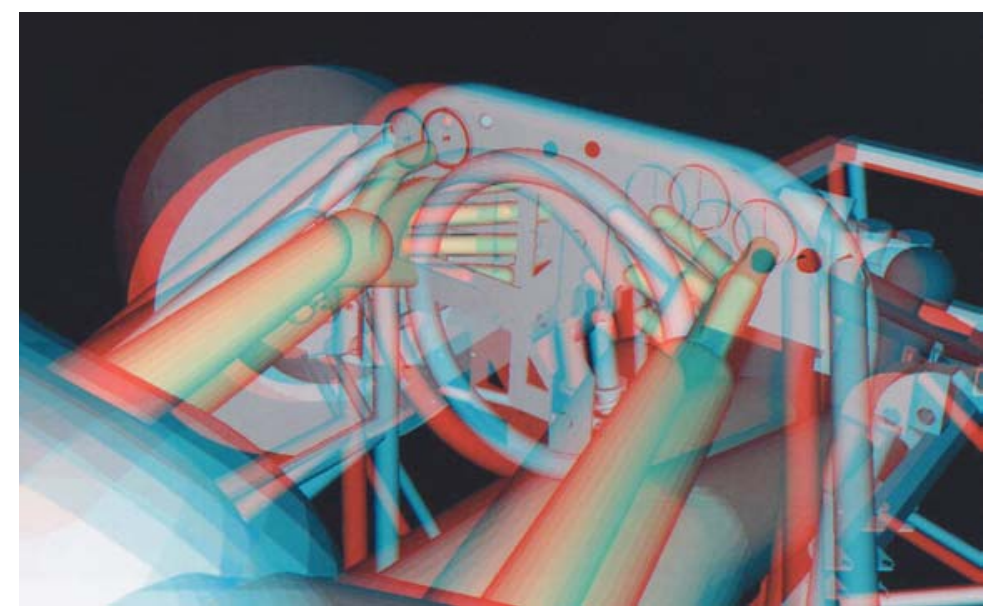

Figure 11. Formula SAE cockpit red-blue anaglyph

\section{Stereo Display}

Use of stereo display technology is a commonly listed component in virtual design, and, in recent years, several immersive CAVE rooms [17] have been installed at Canadian research and educational institutions. To see in stereo, the display alternately renders two eye images. The user wears glasses with active left and right eye LCD shutters [18] that are synchronized with the display. A special display with a $120 \mathrm{~Hz}$ refresh rate is used so that, for each eye, a flicker free [19] $60 \mathrm{~Hz}$ rate is achieved.

Another approach is to separate the left and right eye images using circular polarization or red/blue anaglyphs together with inexpensive passive eyewear.

Direct stereo display remains available off the shelf for mainstream Unix based CAD software, and has in the past been supported for Microsoft Windows NT/2000/XP. A more standard approach is to convert the CAD model Virtual Reality Modeling Language (VRML) [20], and then display it using special purpose viewers. For the projects reported herein, the
Inventor model was converted to a gray level VRML representation using the DynamicDesigner [9] add-on, and then viewed with VCollab [21] running under Windows NT/2000. Shutter glasses, together with a 21 inch monitor, were used for interactive viewing, including "fly-throughs" and moving component animations. Color anaglyh images were also made. Examples for the new home kitchen and the Formula SAE cockpit are shown respectively in Figures 10 and 11.

\section{Online CAD Animation Project}

\section{Motivation}

A natural extension beyond 3D visualization is to include online interaction with the designer. This capability is well established for high priced CAVE systems, but has not been investigated for less expensive CAD systems.

The project undertaken was to develop an online Autodesk Inventor animation capability for a FARO measuring arm. The computer used was a $2.4 \mathrm{GHz}$ 


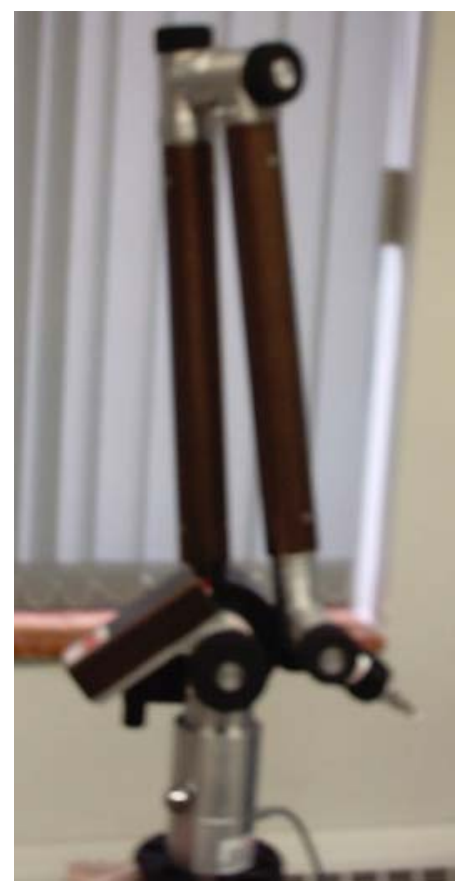

Figure 12. FARO Gold Arm photograph

Pentium IV. Figures 12 and 13 shows a photograph of the FARO arm and the Inventor screen.

The interface used the standard FARO RS232 serial port interface, and communication commands documented in the FARO Gold Software Development Kit (SDK) [22]. Programming was done using the Visual Basic for Applications (VBA) interface provided with Inventor and Microsoft Windows. The initial VBA program logic was based on an infinite loop subroutine that would poll the FARO serial port to obtain the joint angles, and then update the associated Inventor assembly angle constraint variables. More consistent response was later achieved by implementing a standard Windows interval timer with a $200 \mathrm{~ms}$ period. Expiry of the timer triggered an event and corresponding VBA subroutine. The event subroutine again polled the FARO serial port to obtain the joint angles. To increase speed, robotic style link coordinate mathematics [23] specific to the FARO arm were developed, and the arm components were directly positioned ignoring the Inventor constraints. As computer and graphics processing speeds continue to increase, such specific programming should no longer be needed.

A display update rate of $5 \mathrm{~Hz}$ was achieved without the need for additional software optimization. Referring to teleoperation studies, a rate of only 1 or $2 \mathrm{~Hz}$ is adequate [24]. Contact constraint capabilities provided with Inventor could be used together with a haptic workstation [25] (replacing the FARO arm) to create an affordable virtual reality environment.

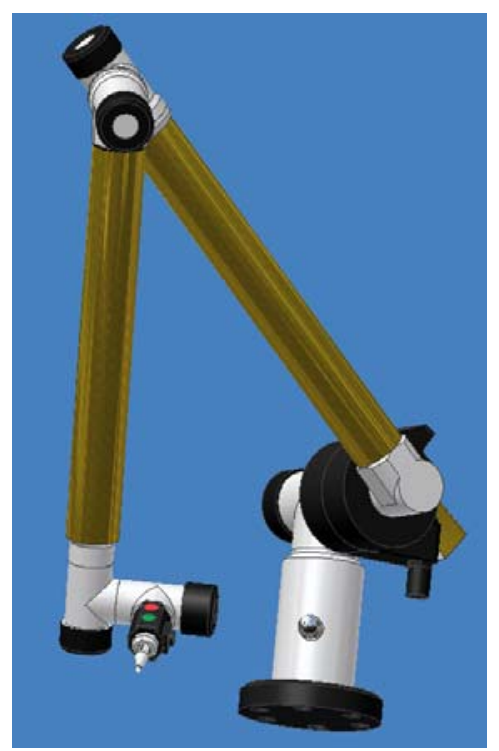

Figure 13. Inventor image of FARO arm

\section{Discussion}

The projects described above illustrate the potential that virtual technology has for undergraduate engineering design projects. Much of the equipment is very affordable, including Windows NT/2000/XP workstations, Microsoft Excel, and Autodesk Inventor. The cost for the two rapid prototype models was approximately CDN\$500. Anaglyph passive stereo eyewear is very inexpensive (less than $\mathrm{CDN} \$ 25$ ), but practically limited to gray images. Active stereo eyewear and a compatible display adapter and monitor is modest in cost (CDN \$4000). The VRML viewing software (e.g. VCollab) is an additional CDN\$2500.

Use of head mounted displays was not considered because of the reported risk developing "motion sickness" [26], or lingering oncular adjustment effects affecting the ability to safety drive or operate equipment immediately afterwards [24]. More recently, eyewear free stereo monitors have appeared [18], and these appear to be a promising alternative.

The usefulness of depth cues for teleoperation has been previously observed [24], and the discontinuation of native shutter glass stereo viewing support in more recent versions of Windows NT/2000/XP solid modeling software is a concern. As stated earlier, for the projects reported herein, VRML files and the VCollab software were used to create stereo images. Unfortunately the translator used does not export texture information from Inventor into the VRML. As well, the extra VRML export step makes it impractical to implement real-time teleoperation with Inventor. The most immediately available solution is to use the animation capability within VRML viewers, or other 
mechanical [27] or architectural digital mockup software. This is a satisfactory approach, since the model is not being updated during teleoperation. However, the contact constraint solver needed for haptic feedback is now lost. In summary, an affordable desktop all-in-one design/stereo render/haptic interface is not yet available. It is noted that several Canadian companies [4][6] are active in virtual design, and hence research investment has return potential.

\section{Summary}

This paper has briefly described the final year McMaster virtual design activities, including three 2003-2004 projects on the development and applications of virtual design tools. The first two projects, on home and automobile design, demonstrated the ability to, using an Excel spreadsheet, quickly choose and update an Inventor based 3D CAD model. The model was then exported into VRML for stereo viewing using the VCollab software. The third project was online CAD animation of a FARO measuring arm, and is the initial step towards implementing a more comprehensive haptic interface. It was observed that an affordable desktop all-in-one design/stereo render/haptic interface system is not available. Software Development Kits (SDKs) available with the separate components can be combined to create such a system, and the effort required to achieve this is currently being investigated.

\section{Acknowledgements}

In addition to B. Ruberto, students participating in the projects were G. Bourne and L. Weinwurm (kitchen), J. Bago, J. Chew and V. Pau (Formula SAE). C. Parsisson implemented the online CAD animation project, and was supported by NSERC and a McMaster University Engineering Undergraduate Research Opportunities Program (UROP) scholarship. Meaghan Jackson created the Solid Edge review part models. Funding for the purchase of the VCollab software, creating the rapid prototype models, and for travel to the conference, was provided by NSERC.

\section{References}

[1] Fleisig, R.V., Robertson, A., and Spence, A.D., "Improving the Spatial Visualization Skills of First Year Engineering Students", Inaugural CDEN Design Conference, Montreal, Quebec, July 29-30, 2004.

[2] Canada Mortgage and Housing Corporation, News Releases, Jan. 9, 2004, www.cmhc.ca.
[3] IKEA, IKEA Kitchen Planner, www.ikea.ca

[4] View22 Technology, Inc., GE Virtual Kitchen, Richmond Hill, ON, www.view22.com.

[5] Microcad Software, Autokitchen, Cresskill, NJ, www.autokitchen.com.

[6] 20-20 Technologies, 20-20 Design, Laval, Québec, www.2020design.com.

[7] Autodesk Inc, AutoCAD, San Rafael, CA www.autodesk.com.

[8] Silicon Graphics, Inc., Mountain View, CA, Open Inventor, http://oss.sgi.com/projects/inventor

[9] Mechanical Dynamics, Inc., DynamicDesigner, Ann Arbor, MI, www.designtechnologies.com.

[10] Ansys, Inc., DesignSpace, Canonsburg, PA, www.ansys.com.

[11] Graphisoft, ArchiCAD, Budapest, Hungary, www.graphisoft.com.

[12] Autodesk Inc, Autodesk Inventor, San Rafael, CA www.autodesk.com.

[13] Ficarra, M., Inventor iMike Anthropomorphic Model, Cross Bros Co. Inc., Rochester, NY, www.xanadu.cz.

[14] Society of Automotive Engineers, 2004 Formula SAE Rules, Warrendale, PA, www.sae.org.

[15] Ashford, F., The Aesthetics of Engineering Design, Business Books Limited, London, UK, 1969.

[16] Damon, A., Stoudt, H., and McFarland, R.A., The Human Body in Equipment Design, Harvard University Press, Cambridge, MA, 1966.

[17] Fakespace Systems, CAVE, Marshalltown, IA, www.fakespace.com.

[18] StereoGraphics Corporation, CrystalEyes and Synthagram, San Rafael, CA, www.stereographics.com.

[19] Canadian Centre for Occupational Health and Safety, Lighting Ergonomics - Light Flicker, www.ccohs.ca.

[20] The VRML Consortium, Inc., Virtual Reality Modeling Language, ISO/IEC 14772-1:1997, www.web3d.org.

[21] VirtualE3D Info. Pvt. Ltd., VCollab, Bangalore, India, www.virtuale3d.com.

[22] FARO Technologies, Inc., FARO Arm Software Development Kit, Lake Mary, FL, Feb. 2000, www.faro.com.

[23] Schilling, R.J., Fundamentals of Robotics - Analysis \& Control, Prentice-Hall, 1990

[24] Liu, A., Tharp, G., French, L., Lai, S., and Stark, L., "Some of What One Needs to Know About Using Head-Mounted Displays to Improve Teleoperator Performance", IEEE Trans. Robotics Automation, 9(5), 1993, pp. 638-648.

[25] Immersion Corp., Haptic Workstation, San Jose, CA, www.3dimmersion.com.

[26] Ebenholtz, S.M., "Motion Sickness and Oculomotors Systems in Virtual Environments", Presence, MIT Press, 1(3), 1992, pp. 302-305.

[27] UGS PLM Solutions, Unigraphics TeamCenter Visualization Mockup, www.ugs.com. 